\title{
Relatively weakly open sets in closed balls of Banach spaces, and real $J B^{*}$-triples of finite rank
}

\author{
Julio Becerra Guerrero • Ginés López Pérez • Antonio M. Peralta • \\ A. Rodríguez-Palacios
}

Received: 13 June 2003 / Revised version: 30 September 2003 /

Published online: 8 June 2004 - (C) Springer-Verlag 2004

\begin{abstract}
We prove that, given a real $J B^{*}$-triple $X$, there exists a nonempty relatively weakly open subset of the closed unit ball of $X$ with diameter less than 2 (if and) only if the Banach space of $X$ is isomorphic to a Hilbert space. Moreover we give the structure of real $J B^{*}$-triples whose Banach spaces are isomorphic to Hilbert spaces. Such real $J B^{*}$-triples are also characterized in two different purely algebraic ways.
\end{abstract}

Mathematics Subject Classification (2000): 46B04, 46B22, 46L05, 46L70

\section{Introduction}

In [25], O. Nygaard and D. Werner discover, perhaps for the first time, how some of the classical Banach spaces which fail the Radon-Nikodym property actually fail to fulfil much weaker requirements. Indeed, it is proved in [25] that, if $X$ is an infinite-dimensional uniform algebra, then $X$ satisfies Property $\mathcal{P}$ which follows:

$(\mathcal{P})$ Every nonempty relatively weakly open subset of the closed unit ball of $X$ has diameter equal to 2 .

This applies in particular to infinite-dimensional real or complex $C(\Omega)$-spaces. We remark that Property $\mathcal{P}$ is fulfilled by every Banach space satisfying the socalled Daugavet property [32, Lemma 3]. Other Banach spaces enjoying Property $\mathcal{P}$ are all infinite-dimensional complex $C^{*}$-algebras, and all $J B$-algebras whose Banach spaces are not isomorphic to Hilbert spaces [3]. The proof that infinitedimensional complex $C^{*}$-algebras satisfy Property $\mathcal{P}$, given in [3], essentially

\footnotetext{
J. BECERRA GUERRERO

Universidad de Granada, Facultad de Ciencias, Departamento de Matemática Applicata, 18071 Granada, Spain (e-mail: juliobg@ugr.es)

G. López Pérez, A. M. Peralta, A. RodríGuez-Palacios

Universidad de Granada, Facultad de Ciencias, Departamento de Análisis Matemático, 18071 Granada, Spain

(e-mail: glopez@ugr.es; aperalta@ugr.es; apalacio@ugr.es)

Partially supported by Junta de Andalucía grant FQM 0199.
} 
relies on the more general fact, also shown in [3], that Property $\mathcal{P}$ is fulfilled by every complex $J B^{*}$-triple whose Banach space is not isomorphic to a Hilbert space. For $J B$-algebras and complex $J B^{*}$-triples the reader is referred to [13] and [18], respectively.

In the present paper we prove as the main result that every real $J B^{*}$-triple $X$ whose Banach space is not isomorphic to a Hilbert space satisfies Property $\mathcal{P}$ (Theorem 2.3). We note that the class of real $J B^{*}$-triples, introduced in [15], contains that of complex $J B^{*}$-triples (regarded as real Banach spaces), as well as that of $J B$-algebras. It is worth mentioning that the proof of Theorem 2.3 provided here is independent of the one given in [3] for complex $J B^{*}$-triples, and has the advantage that it works autonomously in all relevant subclasses of the class of real $J B^{*}$-triples. In fact the key tool in the proof of Theorem 2.3 is that every nonreflexive Banach space $X$ such that $X^{*}$ is an $L$-summand of $X^{* * *}$ fulfils Property $\mathcal{P}$ (Proposition 2.1 ). Thus, since infinite-dimensional complex $C^{*}$-algebras are nonreflexive Banach spaces, and the dual of every complex $C^{*}$-algebra $X$ is an $L$-summand of $X^{* * *}$, we are provided with a $J B^{*}$-triple-free proof of the main result in [3] that infinite-dimensional complex $C^{*}$-algebras $X$ satisfy Property $\mathcal{P}$. By the way, as a consequence of Theorem 2.3, every infinite-dimensional real $C^{*}$-algebra fulfils Property $\mathcal{P}$ (Corollary 2.6).

Theorem 2.3 also contains a precise description of real $J B^{*}$-triples whose Banach spaces are isomorphic to Hilbert spaces. Indeed, such real $J B^{*}$-triples are nothing other than those which can be written as finite $\ell_{\infty}$-sums of simple $J B^{*}$-triples which are either finite-dimensional, infinite-dimensional generalized real spin factors, or of the form $\mathcal{L}(H, K)$ for some real, complex, or quaternionic Hilbert spaces $H, K$ with $\operatorname{dim}(H)=\infty$ and $\operatorname{dim}(K)<\infty$. (Here, for $H$ and $K$ as above, $\mathcal{L}(H, K)$ means the real Banach space of all bounded linear operators from $H$ to $K$.) We complete the description just reviewed by showing two different purely algebraic characterizations of real $J B^{*}$-triples whose Banach spaces are isomorphic to Hilbert spaces. Indeed, they are precisely the real $J B^{*}$ triples of finite rank (Theorem 3.1) as well as those real $J B^{*}$-triples such that all single-generated subtriples are finite-dimensional (Theorem 3.8).

To conclude this introduction, let us note that our results include the generalizations to real $J B^{*}$-triples of the facts proved by Bunce-Chu [7] and Kaup [19] concerning the Radon-Nikodym Property and finite rank, respectively, on complex $J B^{*}$-triples.

\section{The main result}

Let $X$ be a real or complex Banach space. We denote by $S_{X}, B_{X}$, and $X^{*}$ the unit sphere, the closed unit ball, and the (topological) dual, respectively, of $X$. We denote by $w$ the weak topology of $X$, and by $w^{*}$ the weak* topology of $X^{*}$. We always see $X$ as a subspace of its bidual $X^{* *}$ via the canonical injection, and, given 
a subspace $P$ of $X$, we denote by $P^{\circ}$ the polar of $P$ in $X^{*}$. Also, for a bounded linear operator $T$ on $X$, we denote by $T^{*}$ the transpose operator on $X^{*}$. An $L$-projection (respectively, $M$-projection) on $X$ is a linear projection (say $\pi$ ) on $X$ satisfying $\|x\|=\|\pi(x)\|+\|x-\pi(x)\|$ (respectively, $\|x\|=\max \{\|\pi(x)\|,\|x-\pi(x)\|\}$ ) for every $x \in X$. A subspace $P$ of $X$ is said to be an $L$-summand (respectively, $M$-summand) of $X$ if it is the range of an $L$-projection (respectively, $M$-projection) on $X$, and an $M$-ideal of $X$ if $P^{\circ}$ is an $L$-summand of $X^{*}$. The Banach space $X$ is said to be $L$-embedded (respectively, $M$-embedded) whenever $X$ is an $L$-summand (respectively, an $M$-ideal) of $X^{* *}$. According to [14, Proposition III.1.2], $X$ is $M$-embedded if and only if the Dixmier projection on $X^{* * *}$ is an $L$-projection. Consequently, if $X$ is $M$-embedded, then $X^{*}$ is $L$-embedded. The converse is not true. Indeed, in the case that $X$ is a complex $C^{*}$-algebra, $X^{*}$ is always $L$-embedded, whereas $X$ is $M$-embedded if and only if it is a $c_{0}$-sum of algebras of all compact operators on suitable complex Hilbert spaces (see [14]).

Proposition 2.1. Let $X$ be a Banach space such that $X^{*}$ is L-embedded. If there exists a nonempty relatively $w$-open subset of $B_{X}$ with diameter less than 2 , then $X$ is reflexive.

Proof. We have $X^{* * *}=\left(X^{*} \oplus N\right)_{\ell_{1}}$ for some subspace $N$ of $X^{* * *}$, and hence $X^{* * * *}=\left(\left(X^{*}\right)^{\circ} \oplus N^{\circ}\right)_{\ell_{\infty}}$. Assume that there is a nonempty relatively $w$-open subset $U$ of $B_{X}$ with $\operatorname{diam}(U)<2$. Then $U$ contains a set $V$ of the form

$$
\left\{x \in B_{X}:\left|f_{i}\left(x-x_{0}\right)\right|<1 \quad \forall i=1, \ldots, n\right\},
$$

for suitable $x_{0} \in B_{X}, n \in \mathbb{N}$, and $f_{1}, \ldots, f_{n} \in X^{*}$. Put

$$
V^{* *}:=\left\{z \in B_{X^{* *}}:\left|f_{i}\left(z-x_{0}\right)\right|<1 \quad \forall i=1, \ldots, n\right\} .
$$

Since $V^{* *}$ is relatively $w^{*}$-open in $B_{X^{* *}}$, and $B_{X}$ is $w^{*}$-dense in $B_{X^{* *}}$, the set $V$ $\left(=V^{* *} \cap B_{X}\right)$ is $w^{*}$-dense in $V^{* *}$. Therefore $V-V$ is $w^{*}$-dense in $V^{* *}-V^{* *}$, and consequently, by the lower $w^{*}$-semicontinuity of the norm of $X^{* *}$, we have $\operatorname{diam}\left(V^{* *}\right)=\operatorname{diam}(V) \leq \operatorname{diam}(U)<2$. In the same way, the set

$$
V^{* * * *}:=\left\{\beta \in B_{X^{* * * *}}:\left|f_{i}\left(\beta-x_{0}\right)\right|<1 \quad \forall i=1, \ldots, n\right\}
$$

has diameter less than 2. Write $x_{0}=u+v$ with $(u, v) \in\left(X^{*}\right)^{\circ} \times N^{\circ}$. We claim that $B_{\left(X^{*}\right)^{\circ}}+v$ is contained in $V^{* * * *}$. Indeed, for $\alpha \in B_{\left(X^{*}\right)^{\circ}}, \alpha+v$ belongs to $B_{X^{* * * *}}$ because $X^{* * * *}=\left(\left(X^{*}\right)^{\circ} \oplus N^{\circ}\right)_{\ell_{\infty}}$, and, on the other hand, for every $i=1, \ldots, n$ we have $f_{i}\left(\alpha+v-x_{0}\right)=f_{i}(\alpha-u)=0$ because $\left(\alpha-u, f_{i}\right)$ belongs to $\left(X^{*}\right)^{\circ} \times X^{*}$. Keeping in mind that $\operatorname{diam}\left(V^{* * * *}\right)<2$, it follows from the claim just shown that $\operatorname{diam}\left(B_{\left(X^{*}\right)^{\circ}}\right)=\operatorname{diam}\left(B_{\left(X^{*}\right)^{\circ}}+v\right)<2$. Therefore $X$ is reflexive.

We recall that a complex $J B^{*}$-triple is a complex Banach space $X$ with a continuous triple product $\{\ldots\}: X \times X \times X \rightarrow X$ which is linear and symmetric in the outer variables, and conjugate-linear in the middle variable, and satisfies: 
1. For all $x$ in $X$, the mapping $y \rightarrow\{x x y\}$ from $X$ to $X$ is a hermitian operator on $X$ and has nonnegative spectrum.

\section{The main identity}

$$
\{a b\{x y z\}\}=\{\{a b x\} y z\}-\{x\{b a y\} z\}+\{x y\{a b z\}\}
$$

holds for all $a, b, x, y, z$ in $X$.

3. $\|\{x x x\}\|=\|x\|^{3}$ for every $x$ in $X$.

Concerning Condition (1) above, we also recall that a bounded linear operator $T$ on a complex Banach space $X$ is said to be hermitian if $\|\exp (\operatorname{ir} T)\|=1$ for every $r$ in $\mathbb{R}$. Following [15], we define real $J B^{*}$-triples as norm-closed real subtriples of complex $J B^{*}$-triples. Here, by a subtriple we mean a subspace which is closed under triple products of its elements. A triple ideal of a real or complex $J B^{*}$-triple $X$ is a subspace $M$ of $X$ such that $\{X X M\}+\{X M X\} \subseteq M$. We say that the $J B^{*}$-triple $X$ is simple if there are not triple ideals of $X$ others than $\{0\}$ and $X$. Real $J B W^{*}$-triples were first introduced as those real $J B^{*}$-triples which are dual Banach spaces in such a way that the triple product becomes separately $w^{*}$-continuous (see [15, Definition 4.1 and Theorem 4.4]). Later, it was shown in [23] that the requirement of separate $w^{*}$-continuity of the triple product is superabundant. We will apply without notice that the bidual of every real $J B^{*}$-triple $X$ is a $J B W^{*}$-triple under a suitable triple product which extends the one of $X$ [15, Lemma 4.2].

The next proposition becomes the real variant of [2, Proposition 3.4].

Proposition 2.2. The predual of every real $J B W^{*}$-triple is L-embedded.

Proof. Let $X$ be a real $J B W^{*}$-triple, and let $X_{*}$ stand for the predual of $X$. For $x, y \in X$, let $L(x, y)$ and $Q(x, y)$ denote the operators on $X$ defined by $L_{x, y}(z):=\{x y z\}$ and $Q_{x, y}(z):=\{x z y\}$, respectively. By standard theory of duality, the separate $w^{*}$-continuity of the triple product of $X$ is equivalent to the inclusions $\left(L_{x, y}\right)^{*}\left(X_{*}\right) \subseteq X_{*}$ and $\left(Q_{x, y}\right)^{*}\left(X_{*}\right) \subseteq X_{*}$ for all $x, y \in X$. Therefore we have $\left(L_{x, y}\right)^{* *}\left(\left(X_{*}\right)^{\circ}\right) \subseteq\left(X_{*}\right)^{\circ}$ and $\left(Q_{x, y}\right)^{* *}\left(\left(X_{*}\right)^{\circ}\right) \subseteq\left(X_{*}\right)^{\circ}$ for all $x, y \in X$. Keeping in mind the separate $w^{*}$-continuity of the triple product of $X^{* *}$ and the $w^{*}$-density of $X$ in $X^{* *}$, the above inclusions read as $\left\{X X\left(X_{*}\right)^{\circ}\right\} \subseteq\left(X_{*}\right)^{\circ}$ and $\left\{X\left(X_{*}\right)^{\circ} X\right\} \subseteq\left(X_{*}\right)^{\circ}$, respectively. Applying again the separate $w^{*}$-continuity of the triple product of $X^{* *}$ and the $w^{*}$-density of $X$ in $X^{* *}$, we deduce

$$
\left\{X^{* *} X^{* *}\left(X_{*}\right)^{\circ}\right\}+\left\{X^{* *}\left(X_{*}\right)^{\circ} X^{* *}\right\} \subseteq\left(X_{*}\right)^{\circ} .
$$

Therefore $\left(X_{*}\right)^{\circ}$ is a $w^{*}$-closed triple ideal of $X^{* *}$, and hence we have $X^{* *}=\left(X_{*}\right)^{\circ} \oplus P$ for a suitable closed triple ideal $P$ of $X^{* *}$ [15, Lemma 4.3]. Now, the abstract $\ell_{\infty}$-product $\left(\left(X_{*}\right)^{\circ} \times P\right)_{\ell_{\infty}}$ is a real $J B^{*}$-triple in a natural way, and the mapping $\Phi:(u, v) \rightarrow u+v$ from $\left(\left(X_{*}\right)^{\circ} \times P\right)_{\ell_{\infty}}$ to $X^{* *}$ becomes a linear bijection preserving triple products. By [15, Theorem 4.8], $\Phi$ is an isometry, that is $X^{* *}=\left(\left(X_{*}\right)^{\circ} \oplus P\right)_{\ell_{\infty}}$. Thus $\left(X_{*}\right)^{\circ}$ is an $M$-summand of $X^{* *}$, and hence, by [14, Theorem I.1.9], $X_{*}$ is an $L$-summand of $X^{*}$. 
Examples of real $J B^{*}$-triples are the spaces $\mathcal{L}(H, K)$, for arbitrary real, complex, or quaternionic Hilbert spaces $H$ and $K$, under the triple product $\{x y z\}:=$ $\frac{1}{2}\left(x y^{*} z+z y^{*} x\right)$. The above examples become particular cases of those arising by considering either the so-called complex Cartan factors (regarded as real $J B^{*}$-triples) or real forms of complex Cartan factors [20]. We recall that real forms of a complex Banach space $X$ are defined as the real closed subspaces of $X$ of the form $X^{\tau}:=\{x \in X: \tau(x)=x\}$, for some conjugation (i.e., conjugate-linear isometry of period two) on $X$. We note that, if $X$ is a complex $J B^{*}$-triple, then every real form of $X$ is a real $J B^{*}$-triple (since conjugations on $X$ preserve triple products [18]). Among complex Cartan factors, the so-called complex spin factors become specially relevant for our present approach. They are built from an arbitrary complex Hilbert space $(H,(\cdot \mid \cdot))$ of hilbertian dimension $\geq 3$, by taking a conjugation $\sigma$ on $H$, and then by defining the triple product and the norm by

$$
\{x y z\}:=(x \mid y) z+(z \mid y) x-(x \mid \sigma(z)) \sigma(y)
$$

and

$$
\|x\|^{2}:=(x \mid x)+\sqrt{(x \mid x)^{2}-|(x \mid \sigma(x))|^{2}},
$$

respectively, for all $x, y, z$ in $H$. Following [24], we say that a real $J B^{*}$-triple is a generalized real spin factor if it is either a complex spin factor (regarded as a real $J B^{*}$-triple) or a real form of a complex spin factor.

Theorem 2.3. Let $X$ be a real $J B^{*}$-triple. Then the following assertions are equivalent:

1. There exists a relatively $w$-open subset of $B_{X}$ with diameter less than two.

2. The Banach space of $X$ is reflexive.

3. $X$ is a finite $\ell_{\infty}$-sum of closed simple triple ideals which are either finitedimensional, infinite-dimensional generalized real spin factors, or of the form $\mathcal{L}(H, K)$ for some real, complex, or quaternionic Hilbert spaces $H, K$ with $\operatorname{dim}(H)=\infty$ and $\operatorname{dim}(K)<\infty$.

4. The Banach space of $X$ is isomorphic to a Hilbert space.

5. $X$ has the Radon-Nikodym property.

Proof. (1) $\Rightarrow$ (2).- By Proposition 2.2, $X^{*}$ is $L$-embedded. Then Assertion (2) follows from the assumption (1) and Proposition 2.1.

(2) $\Rightarrow$ (3).- By [15, Proposition 2.2], there exists a complex $J B^{*}$-triple $Y$, and a conjugation $\tau$ on $Y$ such that $X=Y^{\tau}$. Since the Banach space of $X$ is reflexive (by the assumption (2)), and $Y=X \oplus i X$, the Banach space of $Y$ is reflexive. Then, by the concluding part of the proof of [7, Proposition 4.5], we have $Y=\left(\oplus_{i=1}^{n} Y_{i}\right)_{\ell_{\infty}}$ where $\left\{Y_{i}\right\}_{i=1, \ldots, n}$ is the family of all minimal triple ideals of $Y$, and moreover, for $i=1, \ldots, n, Y_{i}$ is either finite-dimensional, an infinitedimensional complex spin factor, or of the form $\mathcal{L}(H, K)$ for suitable complex Hilbert spaces $H, K$ with $\operatorname{dim}(H)=\infty$ and $\operatorname{dim}(K)<\infty$. Since $\tau$ preserves 
the triple product of $Y$, and is of period two, the set $\{1, \ldots n\}$ must be the disjoint union of three subsets $A, B, C$ such that $Y_{i}$ is $\tau$-invariant whenever $i$ belongs to $A$, and for each $i \in B$ there is a unique $j \in C$ with $\tau\left(Y_{i}\right)=Y_{j}$. It follows that, putting $Z_{j}:=\left(Y_{j} \oplus \tau\left(Y_{j}\right)\right)_{\ell_{\infty}}$ whenever $j$ lies in $B$, each $Z_{j}$ is $\tau$-invariant, and we have

$$
X=\left(\left(\oplus_{i \in A} Y_{i}^{\tau}\right)_{\ell_{\infty}} \oplus\left(\oplus_{j \in B} Z_{j}^{\tau}\right)_{\ell_{\infty}}\right)_{\ell_{\infty}} .
$$

Since, for $j \in B$, the mapping $y_{j} \rightarrow y_{j}+\tau\left(y_{j}\right)$ from $Y_{j}$ (regarded as a real $J B^{*}$-triple) to $Z_{j}^{\tau}$ is a surjective linear isometry preserving triple products, we can write

$$
X=\left(\left(\oplus_{i \in A} Y_{i}^{\tau}\right)_{\ell_{\infty}} \oplus\left(\oplus_{j \in B} Y_{j}\right)_{\ell_{\infty}}\right)_{\ell_{\infty}} .
$$

Now, to conclude the proof it is enough to show that, if there exists $i \in A$ such that $Y_{i}$ is of the form $\mathcal{L}(H, K)$ for suitable complex Hilbert spaces $H, K$ with $\operatorname{dim}(H)=\infty$ and $\operatorname{dim}(K)<\infty$, then $Y_{i}^{\tau}$ is of the form $\mathcal{L}\left(H^{\prime}, K^{\prime}\right)$ for suitable real or quaternionic Hilbert spaces $H^{\prime}, K^{\prime}$ with $\operatorname{dim}\left(H^{\prime}\right)=\infty$ and $\operatorname{dim}\left(K^{\prime}\right)<\infty$. But this follows from [20, Theorem 4.1].

(3) $\Rightarrow$ (4).- This implication is clear.

(4) $\Rightarrow$ (5) and (5) $\Rightarrow(1)$.- These implications are true even if $X$ is only assumed to be an arbitrary Banach space. Indeed, Banach spaces isomorphic to Hilbert spaces are reflexive, reflexive Banach spaces have the Radon-Nikodym property, the Radon-Nikodym property implies the existence of "slices" of the unit closed ball with arbitrarily small diameter, and such slices are $w$-open relative to the closed unit ball.

For the determination of finite-dimensional simple real $J B^{*}$-triples (including finite-dimensional simple complex $J B^{*}$-triples) and of real forms of complex spin factors, the reader is referred to [22] and [20], respectively.

The next corollary follows straightforwardly from Propositions 2.2 and 2.1, and Theorem 2.3. The Banach space appearing in it could be considered as an arbitrary "nonassociative Lindenstrauss space".

Corollary 2.4. Let $X$ be a real Banach space such that $X^{* *}$ is a real $J B^{*}$-triple (for some triple product). Then there exists a nonempty relatively weakly open subset of the closed unit ball of $X$ with diameter less than 2 (if and) only if $X$ is isomorphic to a Hilbert space.

Let $X$ be a Banach space. For $u$ in $S_{X}$, we define the roughness of $X$ at $u$, $\eta(X, u)$, by the equality

$$
\eta(X, u):=\lim \sup _{\|h\| \rightarrow 0} \frac{\|u+h\|+\|u-h\|-2}{\|h\|} .
$$

We remark that the absence of roughness of $X$ at $u$ (i.e., $\eta(X, u)=0$ ) is nothing other than the Fréchet differentiability of the norm of $X$ at $u$ [10, Lemma 
I.1.3]. Given $\epsilon>0$, the Banach space $X$ is said to be $\epsilon$-rough if, for every $u$ in $S_{X}$, we have $\eta(X, u) \geq \epsilon$. We say that $X$ is rough whenever it is $\epsilon$-rough for some $\epsilon>0$, and extremely rough whenever it is 2-rough.

Invoking the proof of [10, Proposition I.1.11], as is done in the proof of [3, Corollary 2.7], the following corollary follows from Theorem 2.3.

Corollary 2.5. Let $X$ be the predual of a real $J B W^{*}$-triple. Then $X$ is extremely rough if (and only if) it is not isomorphic to a Hilbert space.

Despite real $C^{*}$-algebras can be defined by different systems of intrinsic axioms (see [16] for a summary), we prefer to introduce them as the norm-closed self-adjoint real subalgebras of complex $C^{*}$-algebras. Since complex $C^{*}$-algebras are complex $J B^{*}$-triples under the triple product

$$
\{x y z\}:=\frac{1}{2}\left(x y^{*} z+z y^{*} x\right),
$$

certainly real $C^{*}$-algebras are real $J B^{*}$-triples.

Corollary 2.6. Let $X$ be a real $C^{*}$-algebra. If there exists a non-empty relatively $w$-open subset of $B_{X}$ with diameter less than two, then $X$ is finite dimensional.

Proof. By [28, 4.1.13] and [12, 15.4], there exists a complex $C^{*}$-algebra $Y$ with a conjugate-linear $*$-automorphism $\tau$ of period two such that $X=Y^{\tau}$. Assume that there is a non-empty relativey $w$-open subset of $B_{X}$ with diameter less than two. Then, by Theorem 2.3, the Banach space of $X$ (and hence that of $Y$ ) is reflexive. Finally, apply that complex $C^{*}$-algebras whose Banach spaces are reflexive actually are finite-dimensional [31].

Real $W^{*}$-algebras are usually defined as those real $C^{*}$-algebras which are dual Banach spaces in such a way that the product becomes separately $w^{*}$-continuous (see for instance [8]). Nevertheless, the requirement of separate $w^{*}$-continuity of the product is superabundant [16].

Corollary 2.7. Let $X$ be the predual of a real $W^{*}$-algebra. If $X$ is not extremely rough, then $X$ is finite-dimensional.

\section{Algebraic characterizations of real $J B^{*}$-triples whose Banach spaces are isomorphic to Hilbert spaces}

In this section we are going to prove two purely algebraic characterizations of real $J B^{*}$-triples whose Banach spaces are isomorphic to Hilbert spaces. To this end, it seems to us convenient to introduce the appropriate concepts and basic results at a suitable level of generality. The relevant references in such a level are [17] and [21]. 
From now on, let $\mathbb{F}$ be an arbitrary field of characteristic different from 2 and 3. A Jordan algebra over $\mathbb{F}$ is a (possibly non associative) commutative algebra over $\mathbb{F}$ satisfying the identity $(x y) x^{2}=x\left(y x^{2}\right)$. An element $x$ in a Jordan algebra $X$ with a unit $\mathbf{1}$ is said to be invertible if there exists $y \in X$ such that $x y=\mathbf{1}$ and $x^{2} y=x$. A division Jordan algebra is a nonzero unital Jordan algebra whose nonzero elements are invertible. A Jordan triple over $\mathbb{F}$ is a vector space (say $X$ ) over $\mathbb{F}$ endowed with a TRILINEAR triple product $\{\ldots\}: X \times X \times X \rightarrow X$ satisfying the same main identity required in the definition of complex $J B^{*}$ triples. Now, let $X$ be a Jordan triple over $\mathbb{F}$. A tripotent of $X$ is an element $u \in X$ such that $\{u u u\}=\sigma u$ where $\sigma=\sigma(u)= \pm 1$. Those tripotents $u$ in $X$ satisfying $\sigma(u)=1$ are called positive. Given a tripotent $u$ in $X$, we have $X=X_{0}(u) \oplus X_{1}(u) \oplus X_{2}(u)$, where, for $j \in\{0,1,2\}, X_{j}(u)$ denotes the eigenspace of the operator $x \rightarrow \sigma\{u, u, x\}$ corresponding to the eigenvalue $\frac{1}{2} j$. The space $X_{2}(u)$ becomes a Jordan algebra over $\mathbb{F}$ with unit $u$ under the product $x y:=\sigma\{x u y\}$, and moreover the operator $x \rightarrow \sigma\{u x u\}$ on $X_{2}(u)$ is a linear algebra involution. The tripotent $u$ is said to be a division tripotent whenever $X_{2}(u)$ is a division Jordan algebra. Two tripotents $u, v$ of $X$ are called orthogonal if $u \in X_{0}(v)$, or equivalently $v \in X_{0}(u)$. By a frame in $X$ we mean a family $\mathcal{E}$ of pairwise orthogonal division tripotents of $X$ such that $\bigcap_{u \in \mathcal{E}} X_{0}(u)=0$. We say that $X$ is of finite rank if there exists a finite frame in $X$.

Now, the first main result of this section reads as follows.

Theorem 3.1. Let $X$ be a real $J B^{*}$-triple. Then the Banach space of $X$ is isomorphic to a Hilbert space if and only if $X$ is of finite rank.

The proof of Theorem 3.1 above needs further auxiliary notions and results. Complex $J B^{*}$-algebras are defined as those complete normed Jordan complex algebras $X$ endowed with a conjugate-linear algebra involution $*$ satisfying $\left\|U_{x}\left(x^{*}\right)\right\|=\|x\|^{3}$ for every $x$ in $X$, where, for $x$ in $X$, the operator $U_{x}: X \rightarrow X$ is defined by $U_{x}(y)=2 x(x y)-x^{2} y[34,6,33]$. We define real $J B^{*}$-algebras as the norm-closed self-adjoint real subalgebras of complex $J B^{*}$-algebras. Real $J B^{*}$ algebras were introduced by K. Alvermann [1] (under the name of $J^{*} B$-algebras), who provided a system of intrinsic axioms for them. $J B$-algebras are defined as those complete normed Jordan real algebras $X$ satisfying $\|x\|^{2} \leq\left\|x^{2}+y^{2}\right\|$ for all $x, y \in X$ [13]. Note that, although complex $J B^{*}$-triples are only real Jordan triples, if $u$ is an (automatically positive) tripotent in a complex $J B^{*}$-triple $X$, then the Jordan algebra $X_{2}(u)$ is a complex algebra in a natural way, and the canonical involution of $X_{2}(u)$ becomes conjugate-linear, so that the norm of $X$ converts $X_{2}(u)$ into a complex $J B^{*}$-algebra.

Proposition 3.2. If $K$ is a nonzero real Hilbert space, and if $u$ is any element in $S_{K}$, then the Banach space of $K$, with product and involution defined by

$$
x y:=(x \mid u) y+(y \mid u) x-(x \mid y) u
$$


and

$$
x^{*}:=2(x \mid u) u-x,
$$

respectively, becomes a division real $J B^{*}$-algebra (denoted by $J(K, u)$ ), whose unit is precisely $u$. Moreover, there are no division real $J B^{*}$-algebras others than those constructed above. More precisely, for a nonzero unital real $J B^{*}$-algebra $X$, the following condition are equivalent:

1. $X$ is a division Jordan algebra.

2. The self-adjoint part $X_{\text {sa }}$ of $X$ reduces to $\mathbb{R} \mathbf{1}$.

3. $X=J(K, u)$ for some nonzero real Hilbert space $K$ and some $u \in S_{K}$.

Proof. Let $K$ be a nonzero real Hilbert space. If the dimension of $K$ is 1 or 2, then $J(K, u)$ is $\mathbb{R}$ or $\mathbb{C}$, so that certainly $J(K, u)$ is a division real $J B^{*}$-algebra. Assume that $\operatorname{dim}(K)>2$. Let $H$ denote the hilbertian complexification of $K$, let $\sigma$ stand for the natural conjugation

$$
k_{1}+i k_{2} \rightarrow k_{1}-i k_{2}
$$

on $H$, and let $Y$ be the complex spin factor built from $(H, \sigma)$. Since $u$ is a tripotent in $Y$ with $Y_{2}(u)=Y, Y$ becomes naturally a complex $J B^{*}$-algebra with unit $u$. Since $\sigma$ is a conjugate linear *-automorphism of the complex $J B^{*}$-algebra $Y$, and $J(K, u)=Y^{\sigma}$, we deduce that $J(K, u)$ is a real $J B^{*}$-algebra. Moreover, $J(K, u)$ is a division Jordan algebra because, for $x \in J(K, u) \backslash \mathbb{R} u$, the subalgebra generated by $x$ is a copy of $\mathbb{C}$.

Now, let $X$ be a unital real $J B^{*}$-algebra. We have just proved that Condition (3) in the statement implies Condition (1).

(1) $\Rightarrow(2)$.- As a consequence of [13, Proposition 3.8.2], the self-adjoint part $X_{s a}$ of $X$ is a $J B$-algebra. Assume that (2) does not holds, so that there exists $x \in X_{s a} \backslash \mathbb{R} \mathbf{1}$. Then, since Jordan algebras are power associative [13, Lemma 2.4.5], the closed subalgebra of $X_{s a}$ generated by $x$ and 1 is a unital associative $J B$-algebra, and hence it is of the form $C^{\mathbb{R}}(\Omega)$ for some compact Hausdorff topological space $\Omega$ [13, Theorem 3.2.2], which must have at least two points. Take $y, z \in C^{\mathbb{R}}(\Omega) \backslash\{0\}$ such that $y z=0$. Then we have $U_{y}(z)=0$, which, in view of [17, Theorem I.13.(2)], implies that $y$ is not invertible in $X$, and hence that $X$ is not a Jordan division algebra.

(2) $\Rightarrow(3)$.- Let $X_{s k}$ denote the skew part of $X$. Since the product of two skew elements of $X$ is self-adjoint, the assumption (2) provides us with a symmetric bilinear form $(\cdot \mid \cdot)$ on $X_{s k}$ satisfying $z t=-(z \mid t) 1$ for all $z, t \in X_{s k}$. Since $X=\mathbb{R} \mathbf{1} \oplus X_{s k}$, we can extend $(\cdot \cdot \cdot)$ to a symmetric bilinear form on $X$ by defining $(\mathbf{1} \mid \mathbf{1}):=1$ and $(\mathbf{1} \mid z):=0$ for every $z \in X_{s k}$. Then we have

$$
x y:=(x \mid \mathbf{1}) y+(y \mid \mathbf{1}) x-(x \mid y) \mathbf{1}
$$


and

$$
x^{*}:=2(x \mid \mathbf{1}) \mathbf{1}-x
$$

for all $x, y \in X$. Moreover, since, for $x \in X$, the (automatically self-adjoint) subalgebra of $X$ generated by $x$ is a real $C^{*}$-algebra, and the equality $x^{*} x=(x \mid x) 1$ holds, we deduce that $(\cdot \mid \cdot)$ is an inner product on $X$ satisfying $(x \mid x)=\|x\|^{2}$. Thus the Banach space of $X$ is a Hilbert space (say $K$ ), and we have $X=J(K, \mathbf{1})$.

We note that, if $K$ is a real Hilbert space, and if $u, v$ are in $S_{K}$, then we have $J(K, u)=J(K, v)$ structurally. Indeed, taking a surjective linear isometry $T$ on $K$ with $T(u)=v, T$ becomes an isometric $*$-isomorphism from $J(K, u)$ onto $J(K, v)$. It it also worth mentioning that the algebras $J(K, u)$ have appeared in the literature as the solutions to other problems different from the characterization of division real $J B^{*}$-algebras given by Proposition 3.2. Indeed, the algebras $J(K, u)$ are the unique norm-unital complete normed commutative real algebras whose Banach spaces are smooth at their unit [29]. They are also the unique unital complete normed Jordan real algebras satisfying $\|x\|\left\|x^{-1}\right\|=1$ for every invertible element $x$ [5], as well as the unique norm-unital complete normed commutative real algebras $X$ such that the group of all surjective linear isometries on $X$ acts transitively on $S_{X}$ [4]. Moreover, such algebras become the natural parameters in the construction of all one-sided division complete absolute-valued real algebras [30].

Let $u$ be a tripotent in a real $J B^{*}$-triple $X$. Then the Jordan algebra $X_{2}(u)$, endowed with its natural involution, is a real $J B^{*}$-algebra. Indeed, taking a complex $J B^{*}$-triple $Y$ containing $X$ as a closed real subtriple, $X_{2}(u)$ becomes a closed self-adjoint real subalgebra of the complex $J B^{*}$-algebra $Y_{2}(u)$. We denote by $X^{1}(u)$ the $J B$-algebra of all self-adjoint elements of the real $J B^{*}$-algebra $X_{2}(u)$. With the convention of symbols just made, the following corollary follows from Proposition 3.2.

Corollary 3.3. Let $u$ be a tripotent in a real $J B^{*}$-triple $X$. Then $u$ is a division tripotent if and only if $X^{1}(u)=\mathbb{R} u$.

Corollary 3.4. Let $u$ be a tripotent in a complex $J B^{*}$-triple $X$. Then $u$ is a division tripotent if and only if $X_{2}(u)=\mathbb{C} u$.

Proof. We have $X_{2}(u)=X^{1}(u) \oplus i X^{1}(u)$, and Corollary 3.3 applies.

Thus, in view of Corollary 3.3 (respectively, 3.4), division tripotents in a real (respectively, complex) $J B^{*}$-triple $X$ coincide with the so-called in the literature minimal tripotents of $X$ (see for instance [11] and [19]), which are defined as those tripotents $u$ of $X$ satisfying $X^{1}(u)=\mathbb{R} u$ (respectively, $X_{2}(u)=\mathbb{C} u$ ). The name "minimal" is in agreement with the fact that, in the case that $X$ is actually a 
real or complex $J B W^{*}$-triple, minimal tripotents of $X$ are precisely those tripotents of $X$ which are minimal relative to the order defined in the set of all tripotents of $X$ by $u \leq v$ if and only if $v=u+w$ for some tripotent $w$ orthogonal to $u$ [27, Proposition 2.2]. Now, by arguing as in the proof of [27, Lemma 3.2], we obtain the following corollary.

Corollary 3.5. Let $Y$ be a complex $J B^{*}$-triple with conjugation $\tau$, and let $u$ be a division tripotent of $Y^{\tau}$. Then $u$ is the sum of at most two orthogonal division tripotents of $Y$.

Proof of Theorem 3.1. Assume that the real $J B^{*}$-triple $X$ is of finite rank, so that there exists a finite frame $\left\{u_{1}, \ldots, u_{n}\right\}$ in $X$. Then, taking a complex $J B^{*}$ triple $Y$ with conjugation $\tau$ such that $X=Y^{\tau}$, Corollary 3.5 gives (up to a rearangement, if necessary) the existence of $1 \leq m \leq n$ and division tripotents $v_{1}, \ldots v_{m}, w_{1}, \ldots, w_{m}$ of $Y$ such that $u_{i}=v_{i}+w_{i}$ and $v_{i}$ is orthogonal to $w_{i}$ for $i=1, \ldots, m$, whereas $u_{j}$ is a division tripotent of $Y$ whenever $j=m+1, \ldots, n$. Now it is easily seen that $\left\{v_{1}, \ldots v_{m}, w_{1}, \ldots, w_{m}, u_{m+1}, \ldots, u_{n}\right\}$ is a frame in $Y$, and hence the complex $J B^{*}$-triple $Y$ is of finite rank. By [19, Theorem 4.10], $Y$ is a finite $\ell_{\infty}$-sum of simple triple ideals which are either finite-dimensional, infinitedimensional complex spin factors, or of the form $\mathcal{L}(H, K)$ for complex Hilbert spaces $H, K$ with $\operatorname{dim}(H)=\infty$ and $\operatorname{dim}(K)<\infty$. It follows that the Banach space of $Y$ (and hence that of $X$ ) is isomorphic to a Hilbert space.

To conclude the proof it is enough to show that $X$ is of finite rank whenever the Banach space of $X$ is isomorphic to a Hilbert space. But, in view of the implication (4) $\Rightarrow$ (3) in Theorem 2.3, we are reduced to the case that $X$ is either finite-dimensional, an infinite-dimensional generalized real spin factor, or of the form $\mathcal{L}(H, K)$ for some real, complex, or quaternionic Hilbert spaces $H, K$ with $\operatorname{dim}(H)=\infty$ and $\operatorname{dim}(K)<\infty$. In all these cases, we realize that $X$ is of finite rank by a direct inspection (see [20, Table 1 and Proposition 5.8.(ii)]).

Let $X$ be a Jordan triple over a field $\mathbb{F}$ of characteristic different from 2 and 3 . We say that $X$ is algebraic if all single-generated subtriples of $X$ are finitedimensional over $\mathbb{F}$. If in fact there exists $m \in \mathbb{N}$ such that all single-generated subtriples of $X$ have dimension $\leq m$, then we say that $X$ is of bounded degree, and the minimum such an $m$ will be called the degree of $X$. We define inductively the odd powers of an element $x \in X$ by $x^{1}:=x$ and $x^{2 n+1}:=\left\{x^{2 n-1} x x\right\}$.

Lemma 3.6. Let $X$ be a Jordan triple of degree $m$ over a field $\mathbb{F}$ of characteristic different from 2 and 3 containing at least $2 m+3$ elements. Then every family of nonzero pairwise orthogonal positive tripotents of $X$ has at most $m$ elements.

Proof. Assume on the contrary that we can find nonzero pairwise orthogonal positive tripotents $u_{1}, \ldots, u_{m+1}$ in $X$. Since $\mathbb{F}$ has at least $2 m+3$ elements, we can also find nonzero elements $a_{1}, \ldots, a_{m+1}$ in $\mathbb{F}$ such that $a_{1}^{2}, \ldots, a_{m+1}^{2}$ are pairwise 
different. Put $x:=\sum_{i=1}^{m+1} a_{i} u_{i}$. Since for $j=1, \ldots, m+1$ we have $x^{2 j-1}=$ $\sum_{i=1}^{m+1} a_{i}^{2 j-1} u_{i}$, and the matrix $\left(a_{i}^{2 j-1}\right)_{i, j=1, \ldots, m+1}$ is invertible, the subtriple of $X$ generated by $x$ contains $\left\{u_{1}, \ldots, u_{m+1}\right\}$, and hence is of dimension $\geq m+1$. This contradicts that $X$ is of degree $m$.

We note that Jordan triples are "power-associative". Indeed, for an element $x$ in a Jordan triple $X$, we have $\left\{x^{2 i-1} x^{2 j-1} x^{2 k-1}\right\}=x^{2(i+j+k)-3}$ for all $i, j, k \in \mathbb{N}$, and therefore the subtriple of $X$ generated by $x$ is equal to the linear hull of the set of all odd powers of $x$. Keeping in mind this fact, the proof of [9, Theorem 1], originally made in the setting of complete normed power-associative algebras, works almost verbatim in the setting of complete normed Jordan triples. Thus we have the following lemma.

Lemma 3.7. Let $X$ be an algebraic Jordan triple over $\mathbb{R}$ endowed with a complete norm making the triple product continuous. Then $X$ is of bounded degree.

Now we can prove the concluding main result of the paper.

Theorem 3.8. Let $X$ be a real $J B^{*}$-triple. Then the Banach space of $X$ is isomorphic to a Hilbert space if and only if $X$ is algebraic.

Proof. Assume that $X$ is algebraic. By Lemma 3.7, $X$ is of bounded degree. Let $m$ denote the degree of $X$. We claim that $X^{* *}$ is also of bounded degree (equal to $m$ ). To prove the claim we invoke the so-called strong* (in short $s^{*}$ ) topology of $X^{* *}$ [26, Section 4]. The strong* topology of $X^{* *}$ is a locally convex topology on $X^{* *}$ compatible with the duality $\left(X^{* *}, X^{*}\right)$ [26, Corollary 9], and makes the triple product of $X^{* *}$ jointly continuous on bounded subsets of $X^{* *}$ [26, Theorem 9]. Let $x$ be in $B_{X^{* *}}$. Since $B_{X}$ is $w^{*}$-dense in $B_{X^{* *}}$, the compatibility of $s^{*}$ with the duality $\left(X^{* *}, X^{*}\right)$ gives that $B_{X}$ is also $s^{*}$-dense in $B_{X^{* *}}$, and hence there is a net $\left\{x_{\lambda}\right\}_{\lambda \in \Lambda}$ in $B_{X} s^{*}$-convergent to $x$. Since $X$ is of degree $m$, for $\lambda \in \Lambda$ there exist $a_{0 \lambda}, a_{1 \lambda}, \ldots, a_{m \lambda} \in \mathbb{R}$ such that $\left|a_{0 \lambda}\right|+\left|a_{1 \lambda}\right|+\ldots+\left|a_{m \lambda}\right|=1$ and $a_{0 \lambda} x_{\lambda}+a_{1 \lambda} x_{\lambda}^{3}+\ldots+a_{m \lambda} x_{\lambda}^{2 m+1}=0$. Taking a cluster point $\left(a_{0}, a_{1}, \ldots, a_{m}\right)$ of the net $\left\{\left(a_{0 \lambda}, a_{1 \lambda}, \ldots, a_{m \lambda}\right)\right\}_{\lambda \in \Lambda}$ in $\mathbb{R}^{m+1}$, and applying the joint $s^{*}$-continuity of the triple product of $X^{* *}$ on bounded sets, we obtain $\left|a_{0}\right|+\left|a_{1}\right|+\ldots+\left|a_{m}\right|=1$ and $a_{0} x+a_{1} x^{3}+\ldots+a_{m} x^{2 m+1}=0$. Therefore, the subtriple of $X^{* *}$ generated by $x$ has dimension $\leq m$. Since $x$ is arbitrary in $B_{X^{* *}}$, and $X^{* *}$ contains $X$, and $X$ is of degree $m$, we deduce that $X^{* *}$ is also of degree $m$. Now that the claim is proved, to show that the Banach space of $X$ is isomorphic to a Hilbert space we can assume that $X$ is in fact a $J B W^{*}$-triple of degree $m$. Then, invoking the Krein-Milman theorem, and applying [15, Lemma 3.3], we are provided with a tripotent $u$ in $X$ such that $X_{0}(u)=0$. Let $u_{1}, \ldots, u_{k}$ be nonzero pairwise orthogonal tripotents of $X$ such that $u=u_{1}+\ldots+u_{k}$. According to Lemma 3.6, we must have $k \leq m$. Therefore, we may choose the family $\left\{u_{1}, \ldots, u_{k}\right\}$ above of maximum length, and then each $u_{i}$ becomes a minimal (equivalently, division) tripotent of $X$ (see the 
comments after Corollary 3.4). Now, since $u=u_{1}+\ldots+u_{k}$ and $X_{0}(u)=0$, we have $\cap_{i=1}^{k} X_{0}\left(u_{i}\right)=0$, so $\left\{u_{1}, \ldots, u_{k}\right\}$ is a frame in $X$, and so $X$ is of finite rank. By Theorem 3.1, the Banach space of $X$ is isomorphic to a Hilbert space.

Now assume that the Banach space of $X$ is isomorphic to a Hilbert space. In view of the implication (4) $\Rightarrow$ (3) of Theorem 2.3, to show that $X$ is algebraic we can additionally assume that $X$ is either a generalized real spin factor or of the form $\mathcal{L}(H, K)$ for some real, complex, or quaternionic Hilbert spaces $H, K$ with $\operatorname{dim}(K)<\infty$. Then we realize that $X$ is algebraic by a direct inspection.

Acknowledgements. The authors are grateful to the referee for fruitful suggestions.

\section{References}

[1] Alvermann, K.: Real normed jordan algebras with involution. Arch. Math. 47, 135-150 (1986)

[2] Barton, T. J., Timoney, R. M.: Weak*-continuity of Jordan triple products and applications. Math. Scand. 59, 177-191 (1986)

[3] Becerra, J., López, G., Rodríguez, A.: Relatively weakly open sets in closed balls of $C^{*}$-algebras. J. London Math. Soc. 68, 753-761 (2003)

[4] Becerra, J., López, G., Rodríguez, A.: Isometric reflections on Banach spaces after a paper of A. Skorik and M. Zaidenberg. Rocky Mountain J. Math. 30, 63-83 (2000)

[5] Benslimane, M., Merrachi, N.: Algèbres de Jordan Banach vérifiant $\|x\|\left\|x^{-1}\right\|=1$. J. Algebra 206, 129-134 (1998)

[6] Braun, R. B., Kaup, W., Upmeier, H.: A holomorphic characterization of Jordan $C^{*}$-algebras. Math. Z. 161, 277-290 (1978)

[7] Bunce, L. J., Chu, C. H.: Compact operations, multipliers and Radon-Nikodym property in $J B^{*}$-triples. Pacific J. Math. 153, 249-265 (1992)

[8] Chu, C. H., Dang, T., Russo, B., Ventura, B.: Surjective isometries of real $C^{*}$-algebras. J. London Math. Soc. 47, 87-110 (1993)

[9] Cuartero, B., Galé, J. E.: Bounded degree of algebraic topological algebras. Commun. Algebra 22, 329-337 (1994)

[10] Deville, R., Godefroy, G., Zizler, V.: Smoothness and renormings in Banach spaces. Pitman Monographs and Surveys in Pure and Applied Math. 64, 1993

[11] Friedman, Y., Russo, B.: Structure of the predual of a $J B W^{*}$-triple. J. Reine Angew. Math. 356, 67-89 (1985)

[12] Gooderal, K. R.: Notes on real and complex $C^{*}$-algebras. Shiva Math. Series 5, Devon, 1982

[13] Hanche-olsen, H., Stormer, E.: Jordan operator algebras. Monograph Stud. Math. 21, Pitman, 1984

[14] Harmand, P., Werner, D., Werner, W.: $M$-ideals in Banach spaces and Banach algebras. Lecture Notes in Mathematics 1547, Springer-Verlag, Berlin, 1993

[15] Isidro, J. M., Kaup, W., Rodríguez, A.: On real forms of $J B^{*}$-triples. Manuscripta Math. 86, 311-335 (1995)

[16] Isidro, J. M., Rodríguez, A.: On the definition of real W*-algebras. Proc. Am. Math. Soc. 124, 3407-3410 (1996)

[17] Jacobson, N.: Structure and representations of Jordan algebras. Am. Math. Soc. Coll. Publ. 39, Providence, Rhode Island 1968 
[18] Kaup, W.: A Riemann mapping theorem for bounded symmetric domains in complex Banach spaces. Math. Z. 183, 503-529 (1983)

[19] Kaup, W.: über die Klassifikation der symmetrischen hermiteschen Mannigfaltigkeiten unendlicher Dimension. I. Math. Ann 257, 463-486 (1981)

[20] Kaup, W.: On real Cartan factors. Manuscripta Math. 92, 191-222 (1997)

[21] Loos, O.: Jordan pairs. Lecture Notes in Mathematics 460, Springer-Verlag, Berlin, 1975

[22] Loos, O.: Bounded symmetric domains and Jordan pairs. Mathemathical Lectures, Irvine, University of California at Irvine, 1977

[23] Martínez, J., Peralta, A. M.: Separate weak*-continuity of the triple product in dual real $J B^{*}$-triples. Math. Z. 234, 635-646 (2000)

[24] Moreno, A., Rodríguez, A.: On the Zel'manovian classification of prime $J B^{*}$-triples. J. Algebra 226, 577-613 (2000)

[25] Nygaard, O., Werner, D.: Slices in the unit ball of a uniform algebra. Archiv Math. 76, 441-444 (2001)

[26] Peralta, A. M., Rodríguez, A.: Grothendiekc's inequalities for real and complex $J B W^{*}$ triples. Proc. London Math. Soc. 83, 605-625 (2001)

[27] Peralta, A. M., Stachó, L. L.: Atomic decomposition of real $J B W^{*}$-triples. Quart. J. Math. 52, 79-87 (2001)

[28] Rickart, C. E.: General theory of Banach algebras. Kreiger, New York, 1974

[29] Rodríguez, A.: Nonassociative normed algebras spanned by hermitian elements. Proc. London Math. Soc. 47, 258-274 (1983)

[30] Rodríguez, A.: One-sided division absolute valued algebras. Publ. Mat. 36, 925-954 (1992)

[31] Sakai, S.: Weakly compact operators on operator algebras. Pacific J. Math. 14, 659-664 (1964)

[32] Shvydkoy, R. V.: Geometric aspects of the Daugavet property. J. Funct. Anal. 176, 198-212 (2000)

[33] Youngson, M. A.: Non unital Banach Jordan algebras and $C^{*}$-triple systems. Proc. Edinburgh Math. Soc. 24, 19-31 (1981)

[34] Wright, J. D. M.: Jordan $C^{*}$-algebras. Michigan Math. J. 24, 291-302 (1977) 